\title{
Current-induced phonon renormalization in molecular junctions
}

\author{
Meilin Bai, ${ }^{1,2}$ Clotilde S. Cucinotta, ${ }^{2, *}$ Zhuoling Jiang, ${ }^{1}$ Hao Wang, ${ }^{1}$ Yongfeng Wang, ${ }^{1,3}$ Ivan Rungger, ${ }^{2,4}$ \\ Stefano Sanvito, ${ }^{2, *}$ and Shimin Hou ${ }^{1,3, *}$ \\ ${ }^{1}$ Key Laboratory for the Physics and Chemistry of Nanodevices, Department of Electronics, Peking University, Beijing 100871, China \\ ${ }^{2}$ School of Physics, Advanced Materials and Bioengineering Research Centre (AMBER) and Centre for Research on Adaptive Nanostructures \\ and Nanodevices (CRANN) Institute, Trinity College, Dublin 2, Ireland \\ ${ }^{3}$ Beida Information Research (BIR), Tianjin 300457, China \\ ${ }^{4}$ Materials Division, National Physical Laboratory, Teddington, TW11 0LW, United Kingdom
}

(Received 14 March 2016; revised manuscript received 17 June 2016; published 8 July 2016)

\begin{abstract}
We explain how the electrical current flow in a molecular junction can modify the vibrational spectrum of the molecule by renormalizing its normal modes of oscillations. This is demonstrated with first-principles self-consistent transport theory, where the current-induced forces are evaluated from the expectation value of the ionic momentum operator. We explore here the case of $\mathrm{H}_{2}$ sandwiched between two Au electrodes and show that the current produces stiffening of the transverse translational and rotational modes and softening of the stretching modes along the current direction. Such behavior is understood in terms of charge redistribution, potential drop, and elasticity changes as a function of the current.
\end{abstract}

DOI: 10.1103/PhysRevB.94.035411

\section{INTRODUCTION}

Current-induced forces are at the origin of a rich variety of effects, including vibrations and rotations, as well as mass and energy flow at interfaces [1]. These forces, developing in the presence of a current, underpin electromigration, a phenomenon arising from the transfer of momentum from the conduction electrons to the ions. They can induce structural transformation in nanosystems and transfer energy to phonons, in addition of being the ultimate cause of Joule heating [2]. When the electron current density is large enough, currentinduced forces can control redox reactions at electrode surfaces and affect the functionality and stability of nanodevices, molecular motors, and switches [3-8]. As a consequence, gaining insight into the microscopic mechanisms and achieving control of current-induced forces in nanojunctions will pave the way for key advances in interface chemistry, molecular electronics, and in memory and logic applications.

Molecular electronic devices, consisting of a single or a few molecules bridging two electrodes, have been thoroughly studied [9,10]. In designing robust molecular devices, it is crucial to have information on the status of the molecule in current-carrying conditions, since the applied bias and the current-induced forces may affect the chemical bonds both at the molecule-electrode interfaces and within the molecule itself. Molecular vibrations are directly related to the molecule geometry and inter/intramolecular bonds. Hence, vibrational spectroscopy methods, such as inelastic electron tunneling, infrared, and Raman, have been widely employed to investigate the conduction mechanism and the stability of molecular devices [11-19]. There is also some experimental evidence that the current can modify the vibrational modes of the molecule in a junction [17-19]. For example, the vibrational frequencies of a single $\mathrm{C}_{60}$ sandwiched between two Au electrodes appear to be systematically lowered by the applied bias voltage, indicating that the C-C bonds may be weakened by the current [19].

\footnotetext{
*Corresponding authors: cucinotc@tcd.ie; SANVITOS@tcd.ie; smhou@pku.edu.cn
}

Although experiments may sometimes achieve atomic resolution [20,21], most of them can only measure macroscopic averaged quantities, meaning that the details of the microscopic processes occurring in a nanojunction often remain a matter of conjecture. This implies that computer simulations, which have the power to resolve processes atom by atom, are essential to magnify our view on these phenomena. One way to investigate how a current density perturbs the phonons of a biased system was proposed by Lü et al. [8]. A semiclassical generalized Langevin equation obtained from path integrals was employed together with parameters obtained from density functional theory (DFT). This approach is based on a perturbative expansion of an electron effective action [22] over the electron-phonon interaction matrix. Using this approach, the impact of the different forces-random forces describing Joule heating, current-induced forces including nonconservative wind forces, dissipative frictional forces, and the effective Lorentz-type force due to the Berry phase of the nonequilibrium electrons-was compared for a graphene nanoconstriction carrying high current [23]. The authors observed a strongly nonlinear current-induced heating and a breakdown of the harmonic approximation when the Fermi level was tuned close to a resonance in the electronic structure of the nanoconstriction, caused by the presence of negatively damped phonons.

Here, we take an alternative approach and describe currentinduced forces within the Born-Oppenheimer approximation. Since we are dealing with a nonequilibrium state where the energy may not be conserved [1], we follow the derivation of Di Ventra [24] and compute current-induced forces in a general way from the expectation value of the ionic momentum operator [25]. Importantly, in the presence of a stationary current, charge redistribution occurs, causing atomic rearrangements and modifying the instantaneous Hamiltonian. As a consequence, the vibrational spectrum changes. We account for such phenomena by calculating the electron density with the nonequilibrium Green's function formalism (NEGF) combined with DFT. The density is then used to self-consistently evaluate the effective nonequilibrium 
Hamiltonian and therefore the current-induced forces, the atomic relaxation, and the vibrational spectrum. Compared with previous studies on the phonon spectrum of a molecule under bias [26-28], our calculations appear to have a more rigorous footing, since they explicitly take into account the atomic structure of molecular junctions and possible nonconservative contributions to the forces. Our method is applied to show that the modification of the geometry and the vibrational spectrum of a prototypic $\mathrm{Au}-\mathrm{H}_{2}-\mathrm{Au}$ junction is a genuine effect of the current flow. Eventually, this approach could also be used to perform $a b$ initio molecular dynamics simulations under bias within the Born-Oppenheimer formalism, including the effect of current-induced forces.

\section{CALCULATION METHOD}

Our system consists of a hydrogen molecule sandwiched between two semi-infinite $\mathrm{Au}(100)$ electrodes, with the $\mathrm{H}-\mathrm{H}$ bond parallel to the transport direction. The hydrogen molecule is connected to the electrodes through one small pyramidal gold tip made of a two-atom-long monatomic chain attached to the center of a four-atom square. These four Au atoms are the base of the pyramid and sit on the hollow sites of the $\mathrm{Au}(100)$ surfaces (see Fig. 1). The atomic structure, and vibrational and electronic transport properties of the junction are calculated using the SMEAGOL code [29-31], which is a practical implementation of the NEGF+DFT approach and employs SIESTA as the DFT platform [32]. The atomic cores are described by Troullier-Martins pseudopotentials, and the wave functions of the valence electrons are expanded in terms of atom-centered numerical orbitals [33]. While a double-zeta polarized basis set is used for $\mathrm{H}$, a double-zeta and a single-zeta basis are respectively constructed for the $6 s$ and $5 d / 6 p$ atomic orbitals of Au. Periodic boundary conditions are applied in the plane transverse to the transport, the Au electrodes are constructed with a $(3 \times 3)$ in-plane supercell, and $3 \times 3$ $\mathrm{k}$-points are sampled in the two-dimensional Brillouin zone. The Perdew-Burke-Ernzerhof (PBE) generalized gradient approximation to the exchange and correlation functional is used to account for electron-electron interaction [34]. An equivalent energy cutoff of 800 Ryd is used for the real space integration grid to guarantee an accurate calculation of phonon frequencies. A conjugate gradient geometry relaxation scheme is applied to the junction until all the components of the forces are smaller than $0.01 \mathrm{eV}^{-1}$. With the pseudopotentials and basis functions constructed for the $\mathrm{Au}$ and $\mathrm{H}$ atoms, a lattice constant of $4.18 \AA$ is obtained for bulk gold, which is in fairly

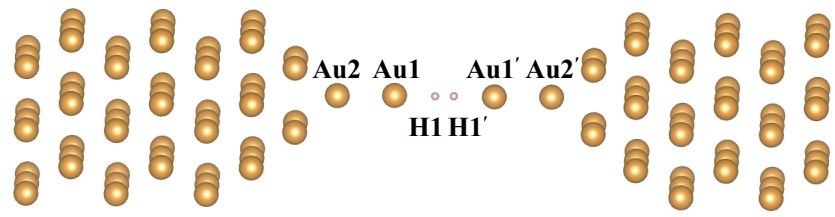

FIG. 1. Optimized atomic structure of the $\mathrm{Au}-\mathrm{H}_{2}-\mathrm{Au}$ molecular junction. The dynamic region for the calculation of the phonon modes includes six atoms, $\mathrm{Au}_{2}, \mathrm{Au}_{1}, \mathrm{H}_{1}, \mathrm{H}_{1^{\prime}}, \mathrm{Au}_{1^{\prime}}$, and $\mathrm{Au}_{2^{\prime}}$. Yellow and white spheres represent $\mathrm{Au}$ and $\mathrm{H}$ atoms, respectively. good agreement with the experimental value of $4.08 \AA$ [35], while the $\mathrm{H}-\mathrm{H}$ bond length and the stretching frequency of the $\mathrm{H}_{2}$ molecule in the gas phase are calculated to be $0.75 \AA$ and $4410 \mathrm{~cm}^{-1}$, respectively. These are both consistent with the experimental values of $0.742 \AA$ and $4395 \mathrm{~cm}^{-1}$ [36,37].

\section{RESULTS AND DISCUSSION}

When the distance between the second-topmost surface layers of the $\mathrm{Au}-\mathrm{H}_{2}-\mathrm{Au}$ junction is set to $22.0 \AA$ (the distance between the electrodes is arbitrary, since in real experiments, it is set by the break junction setup) [38-42], the equilibrium distance between the electrodes' topmost layers is optimized to $17.82 \AA$, and the conductance is $0.74 G_{0}$, in agreement with Ref. [43]. We then characterize the junction under bias by monitoring its geometry, charge redistribution, potential drop, and the energy of the vibrational modes localized on the $\mathrm{H}_{2}$ molecule. The stationary current flow through the junction induced by the applied bias voltage is reflected in geometric modifications. As shown in Fig. 2, under the current flow, the $\mathrm{H}-\mathrm{H}$ bond length becomes progressively larger, while all atoms in the dynamic region, including the two $\mathrm{H}$ atoms and the four neighboring $\mathrm{Au}$ atoms on the left and right sides, consistently move against the current flow $[1,25]$. The electronic signature of the stationary current flow through the junction is a clear charge density polarization, as shown in
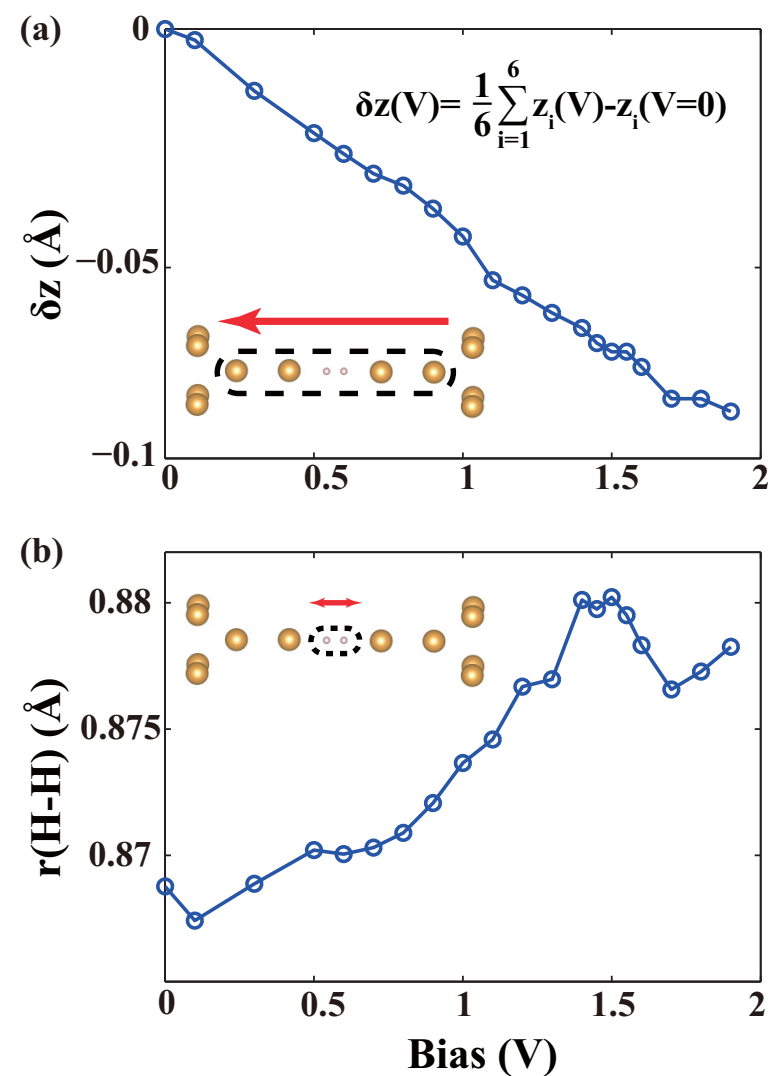

FIG. 2. Geometric signatures of the current flow. (a) Variation of a collective variable, defined by the average shift of the atomic coordinates of the six atoms included in the dynamic region, with the bias. (b) The change of the $\mathrm{H}-\mathrm{H}$ bond length with the bias. 


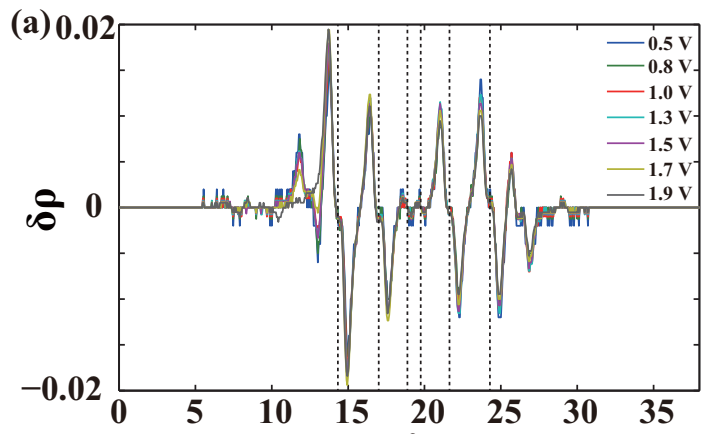

(b)
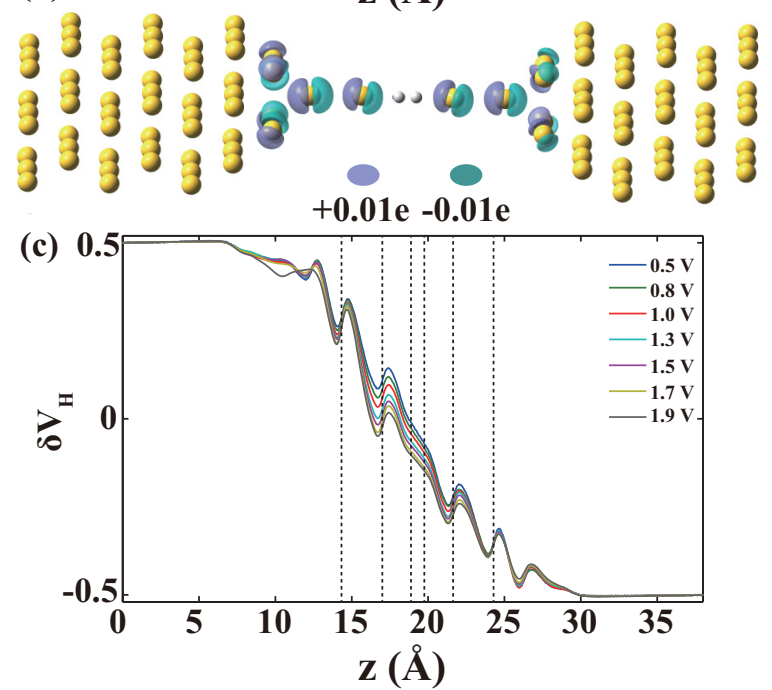

FIG. 3. (a) Planar average of charge density redistribution per unit voltage along the transport direction at different bias voltages, as specified in the legend on the right (in V). (b) Isosurface $(0.018 e$ ) of electron charge density difference between that calculated at 0 and $1.0 \mathrm{~V}$. (c) Planar average of the potential drop per unit voltage along the transport direction at different bias voltages, as specified in the legend on the right (in V). The dotted lines in (a) and (c) indicate the positions of the two $\mathrm{H}$ atoms and their neighboring four $\mathrm{Au}$ atoms.

Fig. 3(b). In the same figure, we also present the average charge density [Fig. 3(a)] and potential drop [Fig. 3(c)] modifications along the current direction. Interestingly, while the curves representing the charge redistribution per unit bias voltage at different biases nearly overlap, the ones representing the potential drop per unit bias voltage are distinct, indicating that the induced electric field is not linearly correlated to the applied bias.

Since the total energy of a current-carrying open system is not necessarily conserved, the dynamical response matrix is calculated by evaluating the first derivative of the currentinduced forces, which are well defined. Such derivatives are computed by finite differences at every applied bias, starting from the relative relaxed geometry and using atomic displacements of $\pm 0.02 \AA$. Phonons are then evaluated by diagonalizing the corresponding dynamical matrix. The $\mathrm{H}_{2}$ molecule and the neighboring four $\mathrm{Au}$ atoms are treated dynamically and yield 18 vibrational modes. Here, we focus on the six localized at $\mathrm{H}_{2}$, which are characterized by much higher frequencies than the others. We also checked that a system with a smaller dynamic region including only two $\mathrm{H}$ atoms returns similar phonons localized on the molecule. However, in order to have a better description of the coupling of the molecule with the electrodes, we use the larger one in the example here. Note that, since a molecular junction is not a periodic system (i.e., it is not translationally invariant), the acoustic phonons sum rule is not applied.

The six vibrational modes localized on the $\mathrm{H}_{2}$ molecule include two parallel modes, namely, one translational (TZ) and one stretching (ST), along the transport direction (the $z$ axis) and four transverse modes. These are the double-degenerate translational modes (TX, TY), in which the center of mass of the $\mathrm{H}_{2}$ molecule vibrates along the transverse directions (the $x$ and $y$ axes), and the double-degenerate rotational modes (RX, RY), in which the angle between the H-H bond and the transport direction vibrates. As we apply a bias, we observe phonon hardening for the transverse translational and rotational modes [see Figs. 4(a) and 4(b)], while the ST mode gets softer [Fig. 4(c)], and the TZ mode remains almost unperturbed [Fig. 4(d)]. We note that the ST mode of the $\mathrm{H}_{2}$ molecule in the junction is much softer than that of $\mathrm{H}_{2}$ in the gas phase. This is consistent with other studies [43,44] and is attributed to the strong interaction of the molecule with the two Au electrodes.

The response of the vibration energies of the two parallel modes to the bias can be related to the system elastic properties, and can be rationalized through a simplified one-dimensional (1D) harmonic oscillator model (see Fig. 5). The oscillator consists of two identical masses, $m$, at positions $x_{1}$ and $x_{2}$, representing the two $\mathrm{H}$ atoms, and three massless springs with the force constants $k, k_{1}$, and $k_{2}$, which connect the two masses to each other and to fixed walls on the left-hand and right-hand sides (representing the electrodes). The equations of motion then simply read

$$
\begin{aligned}
& m \ddot{x}_{1}=-k_{1} x_{1}-k\left(x_{1}-x_{2}\right) \\
& m \ddot{x}_{2}=-k_{2} x_{2}+k\left(x_{1}-x_{2}\right)
\end{aligned}
$$

The model gives a translational mode and a stretching mode along the molecular axis of

$$
\begin{array}{ll}
m \omega_{1}^{2}=f_{1}-f_{2} & \text { (translational) } \\
m \omega_{2}^{2}=f_{1}+f_{2} & \text { (stretching) }
\end{array}
$$

with

$$
\begin{aligned}
& f_{1}=\frac{k_{1}+k_{2}}{2}+k \\
& f_{2}=\sqrt{\left(k_{1}-k_{2}\right)^{2}+4 k^{2}} / 2
\end{aligned}
$$

The model can be mapped onto our NEGF+DFT results because the force constants are easily obtained from the corresponding components of the forces computed with SMEAGOL. Our calculations show that the frequencies obtained by substituting the force constants in Eqs. (2) and (3) at any bias perfectly match those previously calculated with SMEAGOL (see Fig. 4). Hence, the 1D oscillator model is adequate to quantitatively mimic the mechanical behavior of the central region of our junction along the transport direction. 


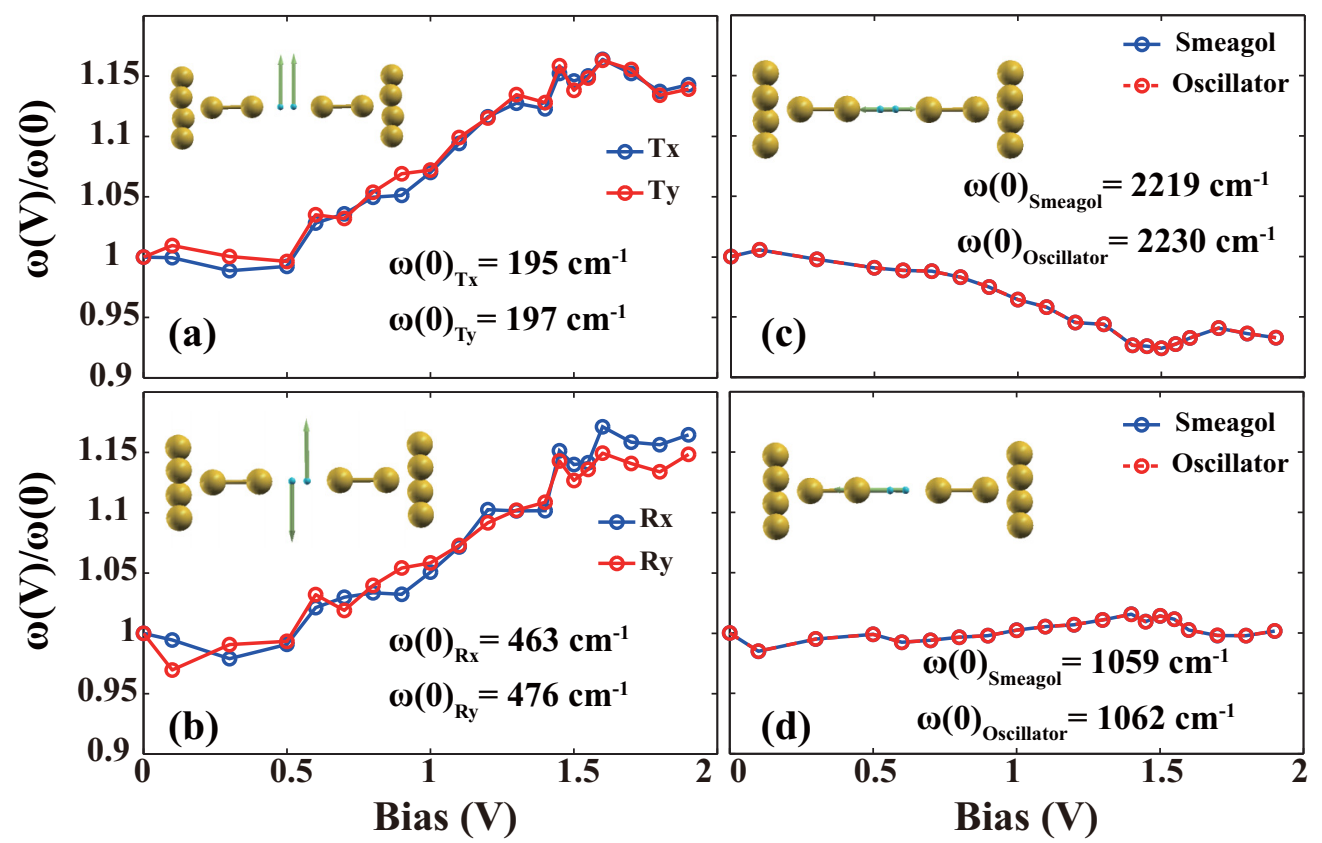

FIG. 4. Relative modifications with the bias of the phonon modes centered on the $\mathrm{H}_{2}$ molecule. (a) Double-degenerate translational modes along the $x$ and $y$ axes. (b) Double-degenerate rotational modes around the $x$ and $y$ axes. (c) Stretching mode along the transport direction. (d) Translational mode along the transport direction. SMEAGOL $=$ spin and molecular electronics algorithm on a generalized atomic orbital landscape.

The force constants calculated from Eq. (1) as a function of the applied bias are plotted in Fig. 5, where it can be seen that $k$ (the force constant between the two $\mathrm{H}$ atoms, mostly responsible for the variation of the stretching mode) quickly decreases with the bias, indicating that the corresponding phonon mode becomes softer. Also $k_{1}$ and $\mathrm{k}_{2}$, connecting $\mathrm{H}_{2}$ to the leads, are affected by the bias. In particular, $k_{1}$ is slightly reduced, whereas $k_{2}$ increases with a slope having an absolute value larger than that of $k_{1}$. Notably, since both $f_{1}$ and $f_{2}$ [see Eq. (3)] decrease with bias, the frequency of the stretching mode ST, obtained as their sum, decreases drastically, whereas the frequency of the parallel translational TZ mode, obtained as their difference, is almost unchanged.

The vibrational behavior of the $\mathrm{Au}-\mathrm{H}_{2}-\mathrm{Au}$ junction results from the complex interplay between the electrostatic

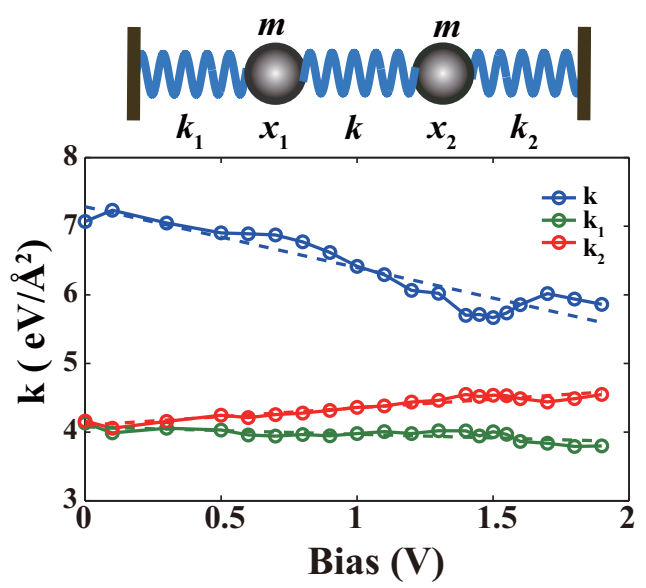

FIG. 5. Top: Model for the 1D harmonic oscillator. Bottom: Force constants versus the applied bias voltage. interaction, the charge redistribution, and the bond strength modifications in the junction. The electrostatic interaction in the junction can be evaluated from a simple Coulomb potential

$$
E_{\mathrm{int}}=\sum_{i<j} \frac{Q_{i} Q_{j}}{4 \pi \varepsilon_{0} r_{i j}},
$$

where $\varepsilon_{0}$ is the vacuum permittivity, $Q_{i}$ and $Q_{j}$ represent the atomic charges (here the Mulliken charges; see Fig. $S 1$ in the Supplemental Material [45]), and $r_{i j}$ represents the interatomic distance between atoms $i$ and $j$. Since no charge redistribution is observed in the electrodes [see Fig. 3(b)], we include in the sum only the atoms belonging to the junction central region, i.e., the gold pyramidal tips and the $\mathrm{H}_{2}$ molecule. We find that the overall electrostatic energy between the hydrogen molecule and the electrodes becomes stronger as the bias voltage gets larger (Fig. S2 in the Supplemental Material [45]). This increase of the molecule-electrode interaction explains the hardening of the transverse rotational and translational modes with the bias.

The softening of the force constant $k$ with bias can be understood by measuring the role of the covalent contribution to the interatomic energy. This can be achieved by analyzing the crystal orbital overlap population (COOP) [46] and the crystal orbital Hamilton population (COHP) [47], accounting for charges of the bonds and associated energies, respectively. In particular, the COOP is the density of states (DOS) weighted by the orbital overlap: The more positive the COOP is, the more bonding character the orbitals possess. Similarly, the COHP is the DOS weighted by the Hamiltonian, which partitions the band-structure energy into orbital-pair interactions, thus measuring the degree of bonding interaction between pairs of atoms. By integrating the $\mathrm{COOP}$ and the $\mathrm{COHP}$ up to the Fermi level, one can evaluate the number of electrons participating in 


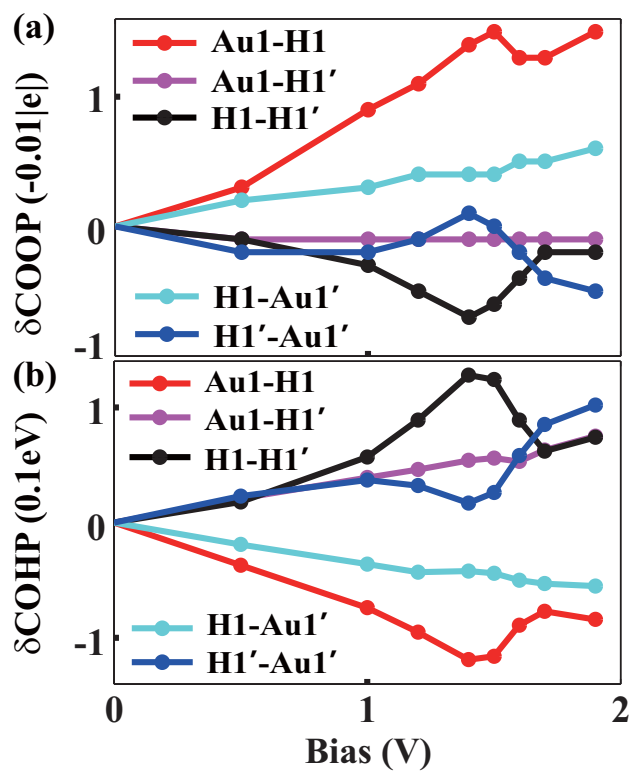

FIG. 6. Changes of the integrals of the (a) COOP and the (b) $\mathrm{COHP}$ at different applied biases with respect to those at zero bias. The pairs of atoms involved are specified in the legends.

a bond and the relative bonding energy, respectively. Usually, the COOP and COHP are evaluated using the coefficients of the molecular orbitals, but these are not explicitly calculated in the NEGF+DFT approach, which computes only the density matrix and the spectral function. Therefore, we extend the concept of COOP and COHP and define both of them between a pair of atoms $I$ and $J$ at the energy $\varepsilon$ in terms of the spectral function, which can be obtained directly from the NEGF+DFT approach [45,48]. These read

$$
\begin{aligned}
\operatorname{COOP}_{I J}(\varepsilon) & =\frac{1}{2 \pi} \sum_{\mu \in I} \sum_{\vartheta \in J} A_{\vartheta \mu}(\varepsilon) S_{\mu \vartheta} \\
\operatorname{COHP}_{I J}(\varepsilon) & =\frac{1}{2 \pi} \sum_{\mu \in I} \sum_{\vartheta \in J} A_{\vartheta \mu}(\varepsilon) H_{\mu \vartheta}
\end{aligned}
$$

where the spectral function $A(\varepsilon)=i\left[G^{\mathrm{R}}(\varepsilon)-G^{\mathrm{A}}(\varepsilon)\right]$ is obtained from the retarded (R) and advanced (A) Green's functions, while $S$ and $H$ are the overlap matrix and the Hamiltonian matrix, respectively. The sums run over all basis orbitals $\mu$ on atom $I$ and $\vartheta$ on atom $J$.

The COOP and COHP for different pairs of atoms in the $\mathrm{Au}-\mathrm{H}_{2}-\mathrm{Au}$ junction are presented in Fig. 6 as a function of the applied bias. Integrals of the COOP and COHP for the two $\mathrm{H}$ atoms up to the Fermi level are calculated to be 0.240 and $-3.896 \mathrm{eV}$, respectively, at zero bias, indicating that the two $\mathrm{H}$ atoms in the $\mathrm{Au}-\mathrm{H}_{2}-\mathrm{Au}$ junction are still in a bonding state. As shown in Fig. 6, as the bias voltage increases, the $\mathrm{H}-\mathrm{H}$ bond progressively acquires a more pronounced antibonding character (more negative COOP and more positive COHP). Such bleaching of the bonding contribution is at the origin of the reduction of the force constant between the two $\mathrm{H}$ atoms, $k$, and hence of the softening of the corresponding stretching mode.
However, this analysis shows that the two $\mathrm{H}$ atoms interact not only with the immediately adjacent $\mathrm{Au}$ atoms $\left(\mathrm{Au}_{1}-\mathrm{H}_{1}\right.$ and $\mathrm{Au}_{1^{\prime}}-\mathrm{H}_{1^{\prime}}$, respectively; see Fig. 1 for the atom numbering), but also with their second neighbors $\left(\mathrm{Au}_{1}-\mathrm{H}_{1^{\prime}}\right.$ and $\mathrm{Au}_{1^{\prime}}-\mathrm{H}_{1}$, respectively). A modulation of the bonding strength of neighboring pairs of atoms is observed, alternatively becoming stronger and weaker. As a result, unraveling the trends of force constants $k_{1}$ and $k_{2}$ is not trivial. Partitioning the electrostatic energy into the contributions from $\mathrm{H}_{1}$ and from $\mathrm{H}_{1^{\prime}}$ to all the $\mathrm{Au}$ atoms helps in understanding the aforementioned asymmetry in the slopes of $k_{2}$ and $k_{1}$. As shown in Fig. S2 in the Supplemental Material [45], these two contributions to the electrostatic interaction become stronger (the energy becomes more negative) with bias, but this effect is more pronounced for the interaction involving the $\mathrm{H}_{1^{\prime}}$ atom (the curve of $\mathrm{H}_{1^{\prime}}-\mathrm{Au}_{\text {all }}$ is always below the $\mathrm{H}_{1}-\mathrm{Au}_{\text {all }}$ curve). This enhanced electrostatic interaction increases the slope of $k_{2}$, thus explaining the asymmetry.

The opposite trends of $k_{1}$ and $k_{2}$ with the bias are also reflected in a charge depletion and accumulation in the regions on the left-hand side and on the right-hand side of the $\mathrm{H}_{2}$ molecule (see Fig. S2 in the Supplemental Material [45]). However, since opposite contributions to the interatomic bonding energy sum up in a nontrivial way, such charge depletion and accumulation along $\mathrm{Au}_{1}-\mathrm{H}_{1}$ and $\mathrm{Au}_{1^{\prime}}-\mathrm{H}_{1^{\prime}}$ (see the right panel of Fig. S2 in the Supplemental Material [45]) do not produce weakening and strengthening of these bonds. The opposite trend is rather observed (see $\mathrm{Au}_{1}-\mathrm{H}_{1}$ and $\mathrm{Au}_{1^{\prime}}-\mathrm{H}_{1^{\prime}}$ curves in Fig. 6). Thus, these two numbers $k_{1}$ and $k_{2}$, describing a progressively weaker and stronger coupling with the electrodes, sum up at each bias all the different interactions between the $\mathrm{H}_{1}$ and $\mathrm{H}_{1^{\prime}}$ atoms and the other $\mathrm{Au}$ atoms on the left-hand and right-hand sides of the junction.

The atomic forces in our calculations include both currentinduced and field-induced components. In order to establish which force is at the origin of the softening of the stretching mode with the applied bias, we simulated an isolated hydrogen molecule in a uniform electric field (Table I). In agreement with previous studies [49-52], we observed that the electric field makes the $\mathrm{H}-\mathrm{H}$ bond longer and redshifts the stretching frequency, as also observed in the $\mathrm{Au}-\mathrm{H}_{2}-\mathrm{Au}$ junction. By evaluating the intensity of the equivalent electric field experienced by the $\mathrm{H}_{2}$ molecule in the junction at a given applied bias, as obtained by computing the Hartree potential drop in the region of the two hydrogen atoms, we find fields in the range $0.21-1.0 \times 10^{9} \mathrm{~V} \mathrm{~m}^{-1}$ for biases between $0.1 \mathrm{~V}$ and $2.0 \mathrm{~V}$. However, these are too weak to affect the bond length, the charge redistribution, and the stretching frequency. This can be seen in Table I, which shows that the bond length and the stretching mode of a hydrogen molecule start to be affected

TABLE I. Effects of the applied electric field $\left(F\right.$, in $\left.\times 10^{10} \mathrm{~V} \mathrm{~m}^{-1}\right)$ on the $\mathrm{H}-\mathrm{H}$ bond length $(r$, in $\AA$ ) and the frequency of the stretching mode $\left(\omega\right.$, in $\left.\mathrm{cm}^{-1}\right)$ of an isolated $\mathrm{H}_{2}$ molecule.

\begin{tabular}{llllllllll}
\hline \hline$F$ & 0.000 & 0.025 & 0.050 & 0.100 & 0.200 & 0.500 & 1.000 & 2.000 & 5.000 \\
$r$ & 0.750 & 0.750 & 0.750 & 0.750 & 0.750 & 0.751 & 0.752 & 0.757 & 0.802 \\
$\omega$ & 4411 & 4410 & 4410 & 4409 & 4410 & 4406 & 4377 & 4272 & 3441 \\
\hline \hline
\end{tabular}


by the electric field for fields at least one order of magnitude larger than that experienced by the molecule in the junction. As a consequence, the evolution under bias of the vibrational frequencies is mainly determined by the current flow, rather than by the electric field. Moreover, the ratio between the applied bias and the equivalent electric field experienced by the $\mathrm{H}_{2}$ molecule in the junction becomes larger at higher bias. Since the junction conductance changes little with the bias (see Fig. S3 in the Supplemental Material [45]), a resistance decrease between the two hydrogen atoms is implied.

\section{CONCLUSION}

We used the NEGF+DFT approach to evaluate currentinduced forces and thus the phonon spectrum of the prototypic $\mathrm{Au}-\mathrm{H}_{2}-\mathrm{Au}$ molecular junction under bias. We observed an elongation of the $\mathrm{H}-\mathrm{H}$ bond length and a shift against the current flow of the central dynamic region of the junction. The most important signature of the current flow is the stiffening of the $\mathrm{H}_{2}$ transverse translational and rotational modes and the softening of the stretching mode along the current direction. The stiffening of the transverse modes is due to an increase with bias of the overall electrostatic interaction, and the softening of the stretching mode is due to the enhanced antibonding nature of the $\mathrm{H}-\mathrm{H}$ bond, as rationalized through a simple 1D oscillator model. Finally, by analyzing the effective electric field experienced by the $\mathrm{H}_{2}$ molecule in the junction, we conclude that the observed geometrical and vibrational changes are dominated by the electric current rather than by the electric field. Our work shows that current-induced forces can affect the structure and the stability of molecular electronic devices, and this can be captured in a simple and elegant way by this methodology.

\section{ACKNOWLEDGMENTS}

This project was supported by the National Natural Science Foundation of China (Grants No. 61321001 and No. 61271050) and the Ministry of Science and Technology of China (Grant No. 2013CB933404). S.S. acknowledges additional funding support from the European Research Council ("quantitative electron and spin transport theory for organic crystals based devices" QUEST project, Project No. 307891). I.R. thanks the European Union for financial support through the FP7 Project No. 618082 ACMOL. Computational resources were provided by the Trinity Centre for High Performance Computing (TCHPC) and the Irish Centre for High-End Computing (ICHEC).
[1] T. N. Todorov, D. Dunda, J.-T. Lü, M. Brandbyge, and P. Hedegård, Eur. J. Phys. 35, 065004 (2014).

[2] J.-T. Lü, M. Brandbyge, and P. Hedegård, Nano Lett. 10, 1657 (2010).

[3] N. Bode, S. V. Kusminskiy, R. Egger, and F. von Oppen, Phys. Rev. Lett. 107, 036804 (2011).

[4] J. Brüggemann, G. Weick, F. Pistolesi, and F. von Oppen, Phys. Rev. B 85, 125441 (2012).

[5] D. Dundas, E. J. McEniry, and T. N. Todorov, Nat. Nanotechnol. 4, 99 (2009).

[6] H. L. Tierney, C. J. Murphy, A. D. Jewell, A. E. Baber, E. V. Iski, H. Y. Khodaverdian, A. F. McGuire, N. Klebanov, and E. C. H. Sykes, Nat. Nanotechnol. 6, 625 (2011).

[7] R. Bustos-Marún, G. Refael, and F. von Oppen, Phys. Rev. Lett. 111, 060802 (2013).

[8] J.-T. Lü, M. Brandbyge, P. Hedegård, T. N. Todorov, and D. Dundas, Phys. Rev. B 85, 245444 (2012).

[9] N. J. Tao, Nat. Nanotechnol. 1, 173 (2006).

[10] A. Nitzan and M. A. Ratner, Science 300, 1384 (2003).

[11] J. Hihath and N.J. Tao, Prog. Surf. Sci. 87, 189 (2012).

[12] N. Okabayashi, M. Paulsson, and T. Komeda, Prog. Surf. Sci. 88, 1 (2013).

[13] Y. Jun and X.-Y. Zhu, J. Am. Chem. Soc. 126, 13224 (2004).

[14] A. Jaiswal, K. G. Tavakoli, and S. Zou, Anal. Chem. 78, 120 (2006).

[15] Z. Ioffe, T. Shamai, A. Ophir, G. Noy, I. Yutsis, K. Kfir, O. Cheshnovsky, and Y. Selzer, Nat. Nanotechnol. 3, 727 (2008).

[16] D. R. Ward, N. J. Halas, J. W. Ciszek, J. M. Tour, Y. Wu, P. Nordlander, and D. Natelson, Nano Lett. 8, 919 (2008).

[17] D. R. Ward, D. A. Corley, J. M. Tour, and D. Natelson, Nat. Nanotechnol. 6, 33 (2011).

[18] Z. Liu, S.-Y. Ding, Z.-B. Chen, X. Wang, J.-H. Tian, J. R. Anema, X.-S. Zhou, D.-Y. Wu, B.-W. Mao, X. Xu, B. Ren, and Z.-Q. Tian, Nat. Commun. 2, 305 (2011).
[19] Y. Li, P. Doak, L. Kronik, J. B. Neaton, and D. Natelson, Proc. Natl. Acad. Sci. USA 111, 1282 (2014).

[20] B. C. Stipe, M. A. Rezaei, and W. Ho, Science 279, 1907 (1998).

[21] L. J. Lauhon and W. Ho, Phys. Rev. Lett. 85, 4566 (2000).

[22] M. Brandbyge, P. Hedegård, T. F. Heinz, J. A. Misewich, and D. M. Newns, Phys. Rev. B 52, 6042 (1995).

[23] T. Gunst, J.-T. Lü, P. Hedegård, and M. Brandbyge, Phys. Rev. B 88, 161401(R) (2013).

[24] M. Di Ventra, Electrical Transport in Nanoscale Systems (Cambridge University Press, Cambridge, United Kingdom, 2008).

[25] R. Zhang, I. Rungger, S. Sanvito, and S. Hou, Phys. Rev. B 84, 085445 (2011).

[26] N. Sergueev, D. Roubtsov, and H. Guo, Phys. Rev. Lett. 95, 146803 (2005).

[27] F. Mirjani, J. M. Thijssen, and M. A. Ratner, J. Phys. Chem. C 116, 23120 (2012).

[28] K. Kaasbjerg, T. Novotný, and A. Nitzan, Phys. Rev. B 88, 201405 (2013).

[29] A. R. Rocha, V. M. García-Suárez, S. W. Bailey, C. J. Lambert, J. Ferrer, and S. Sanvito, Nat. Mater. 4, 335 (2005).

[30] A. R. Rocha, V. M. García-Suárez, S. Bailey, C. Lambert, J. Ferrer, and S. Sanvito, Phys. Rev. B 73, 085414 (2006).

[31] I. Rungger and S. Sanvito, Phys. Rev. B 78, 035407 (2008).

[32] J. M. Soler, E. Artacho, J. D. Gale, A. García, J. Junquera, P. Ordejón, and D. Sánchez-Portal, J. Phys.: Condens. Matter 14, 2745 (2002).

[33] N. Troullier and J. L. Martins, Phys. Rev. B 43, 1993 (1991).

[34] J. P. Perdew, K. Burke, and M. Ernzerhof, Phys. Rev. Lett. 77, 3865 (1996).

[35] C. Kittel, Introduction to Solid State Physics, 8th ed. (Wiley, Hoboken, NJ, 2004). 
[36] P. C. H. Mitchell, P. Wolohan, D. Thompsett, and S. J. Cooper, J. Mol. Catal. A: Chem. 119, 223 (1997).

[37] D. A. Mcquarrie and J. D. Simon, Physical Chemistry: A Molecular Approach (University Science Books, Sausalito, CA, 1997).

[38] M. Kiguchi, T. Nakazumi, K. Hashimoto, and K. Murakoshi, Phys. Rev. B 81, 045420 (2010).

[39] T. Nakazumi, S. Kaneko, and M. Kiguchi, J. Phys. Chem. C 118, 7489 (2014).

[40] Y. Li, S. Kaneko, S. Fujii, and M. Kiguchi, J. Phys. Chem. C 119, 19143 (2015).

[41] S. Li, A. Yu, F. Toledo, Z. Han, H. Wang, H. Y. He, R. Wu, and W. Ho, Phys. Rev. Lett. 111, 146102 (2013).

[42] H. Wang, S. Li, H. He, A. Yu, F. Toledo, Z. Han, W. Ho, and R. Wu, J. Phys. Chem. Lett. 6, 3453 (2015).

[43] T. Frederiksen, M. Paulsson, and M. Brandbyge, J. Phys.: Conf. Ser. 61, 312 (2007).

[44] R. N. Barnett, H. Häkkinen, A. G. Scherbakov, and U. Landman, Nano Lett. 4, 1845 (2004).
[45] See Supplemental Material at http://link.aps.org/supplemental/ 10.1103/PhysRevB.94.035411 for details of the Mulliken charge, electrostatic energy, and I-V characteristics of the $\mathrm{Au}-\mathrm{H}_{2}-\mathrm{Au}$ molecular junction, and the derivation of COOP and COHP in transport systems.

[46] T. Hughbanks and R. Hoffmann, J. Am. Chem. Soc. 105, 3528 (1983).

[47] R. Dronskowski and P. E. Blöchl, J. Phys. Chem. 97, 8617 (1993).

[48] M. Brandbyge, K. Stokbro, J. Taylor, J.-L. Mozos, and P. Ordejón, Phys. Rev. B 67, 193104 (2003).

[49] H. Nakatsuji, T. Hayakawa, and T. Yonezawa, J. Am. Chem. Soc. 103, 7426 (1981).

[50] J. L. Andrés, J. Martí, M. Duran, A. Lledós, and J. Bertrán, J. Chem. Phys. 95, 3521 (1991).

[51] M. Duran, J. L. Andrés, A. Lledós, and J. Bertrán, J. Chem. Phys. 90, 328 (1989).

[52] W. Gründler, T. Steinke, and P. Walther, J. Comput. Chem. 11, 548 (1990). 